\title{
Nonrigid Shape Correspondence Using Landmark Sliding, Insertion and Deletion
}

\author{
Theodor Richardson and Song Wang \\ Department of Computer Science and Engineering, \\ University of South Carolina, \\ Columbia, SC 29208, USA \\ \{richa268, songwang\}@cse.sc.edu
}

\begin{abstract}
The growing usage of statistical shape analysis in medical imaging calls for effective methods for highly accurate shape correspondence. This paper presents a novel landmark-based method to correspond a set of $2 \mathrm{D}$ shape instances in a nonrigid fashion. Different from prior methods, the proposed method combines three important factors in measuring the shape-correspondence error: landmark-correspondence error, shape-representation error, and shape-representation compactness. In this method, these three important factors are explicitly handled by the landmark sliding, insertion, and deletion operations, respectively. The proposed method is tested on several sets of structural shape instances extracted from medical images. We also conduct an empirical study to compare the developed method to the popular Minimum Description Length method.
\end{abstract}

\section{Introduction}

Most anatomical structures posses a unique shape, which plays a critical role in modern medical image analysis. Statistical shape analysis (SSA) 258] is a very powerful tool for identifying and representing the underlying shape information of a certain structure. Particulary, SSA can construct a statistical shape model, usually from a set of individual shape instances, to describe the deformation space of the underlying shape. In 2D cases, each shape instance is in the form of a continuous curve. For convenience, we refer to this ground-truth continuous form of a shape instance as a shape contour.

Many researchers 63. have pointed out that the accuracy of the shape correspondence greatly affects the accuracy of SSA. In addition, most current SSA methods operate on a set of sparsely sampled landmarks along the shape contours. Therefore, our goal of shape correspondence is to find a way to locate a small set of corresponded landmarks along each shape instance. As in most SSA methods, landmarks discussed in this paper refer to a set of sampled points along the shape instances and may not coincide with anatomically critical points.

For the landmark-based shape correspondence, the following three important factors are critical in order to correctly model the shape-correspondence 
error: landmark-correspondence error, representation error, and representation compactness. In general, a small shape-correspondence error implies a small landmark-correspondence error, a small representation error, and a high representation compactness. Landmark-correspondence error should be small because we use it as an approximation of the underlying shape-correspondence error. This is a factor that has been widely considered in most prior shape-correspondence methods 4,16]. Small representation error is also important because the above approximation is accurate only when the sampled landmarks well represent the underlying shape contour. High representation compactness means that the landmark sampling should be as sparse as possible, which is desired in most SSA methods. Clearly, small representation error and high representation compactness are contradictory and require a balance.

With a set of roughly-corresponded landmarks, Bookstein [3] presents an algorithm to move these landmarks along the tangent directions of the shape contour to achieve a minimum landmark correspondence error that is defined by the thin-plate bending energy. However, the resultant landmarks may not be located on the underlying shape contour and, therefore, the representation error may be large. Wang, Kubota, and Richardson [1] address this problem by adding a step of projecting the landmarks back to the shape contour. This, however, is still not sufficient to achieve small representation error. Representation compactness is not considered in either of these two methods. Minimum Description Length (MDL) 6] is arguably the state-of-the-art method for landmark-based shape correspondence. The shape-correspondence error in MDL is measured by the required bit-length to transmit these shape instances. Genetic algorithms are usually used to locate the optimal landmarks in MDL. Recent efforts have been made to incorporate the factor of representation error into MDL [109].

In this paper, we develop a new method to explicitly consider the three factors listed above. More specifically, the proposed method combines three operations: landmark sliding, landmark insertion, and landmark deletion, which explicitly address the above three factors, respectively.

\section{Problem Formulation}

In this paper, we consider $2 \mathrm{D}$ shape correspondence, i.e., each shape instance is in the form of a shape contour, which can be open or closed. For simplification, we describe the shape-correspondence algorithm based on closed-curve shape instances, which can be easily extended to deal with open-curve shapes. Denote the given set of closed-curve shape instances to be $S=\left\{S_{1}, S_{2}, \ldots, S_{n}\right\}$. Each shape instance $S_{i}$ is in the form of an arc-length parameterized curve $\mathbf{s}_{i}\left(t_{i}\right)=$ $\left(x_{i}\left(t_{i}\right), y_{i}\left(t_{i}\right)\right), 0 \leq t_{i} \leq L_{i}$, where $L_{i}$ is the perimeter of $S_{i}$ and $t_{i}$ is the traversed curve length from $\mathbf{s}_{i}(0)$ to $\mathbf{s}_{i}\left(t_{i}\right)$. The goal of the landmark-based shape correspondence is to identify the same number $m$ landmarks $\mathbf{s}_{i}\left(t_{i 1}\right), \mathbf{s}_{i}\left(t_{i 2}\right), \ldots, \mathbf{s}_{i}\left(t_{i m}\right)$ along each shape instance $S_{i}$ such that for any $k$, the $n$ landmarks $\mathbf{s}_{i}\left(t_{i k}\right)$, $i=1,2, \ldots, n$, are corresponded across these $n$ shape instances. For brevity, 
we denote $\mathbf{v}_{i k}=\mathbf{s}_{i}\left(t_{i k}\right)$ as a landmark and $V_{i}=\left\{\mathbf{v}_{i 1}, \mathbf{v}_{i 2}, \ldots, \mathbf{v}_{i m}\right\}$ to be the sampled landmark set along $S_{i}$. For convenience, we further assume that the $m$ landmarks in $V_{i}$ are sampled sequentially along $S_{i}$, as shown in Fig. 11(a).

As mentioned above, we need to define the measures for landmarkcorrespondence error, representation error, and representation compactness to fully model the underlying shape-correspondence error. For representation compactness, the measure is simply the number of landmarks $m$, i.e., we desire the number $m$ to be as small as possible. The representation error measures the error of using these $m$ landmarks to represent the underlying shape contour. As shown in Fig. 1(a), let $R\left(V_{i}, S_{i}\right)$ be the total discrepancy area (shown as dark gray regions) between the underlying shape contour $S_{i}$ and the polygon formed by sequentially connecting the $m$ landmarks in $V_{i}$ for shape instance $i$. We define representation error as

$$
\alpha\left(V_{i}, S_{i}\right)=\frac{R\left(V_{i}, S_{i}\right)}{R\left(S_{i}\right)},
$$
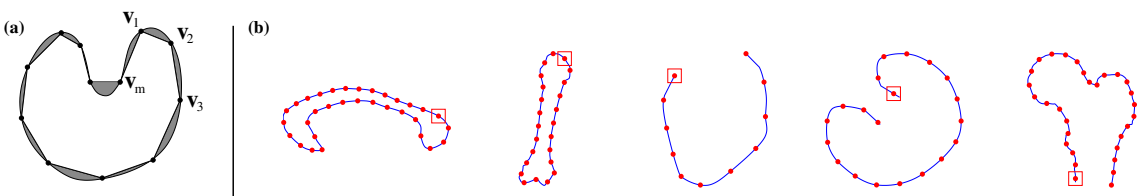

(c)
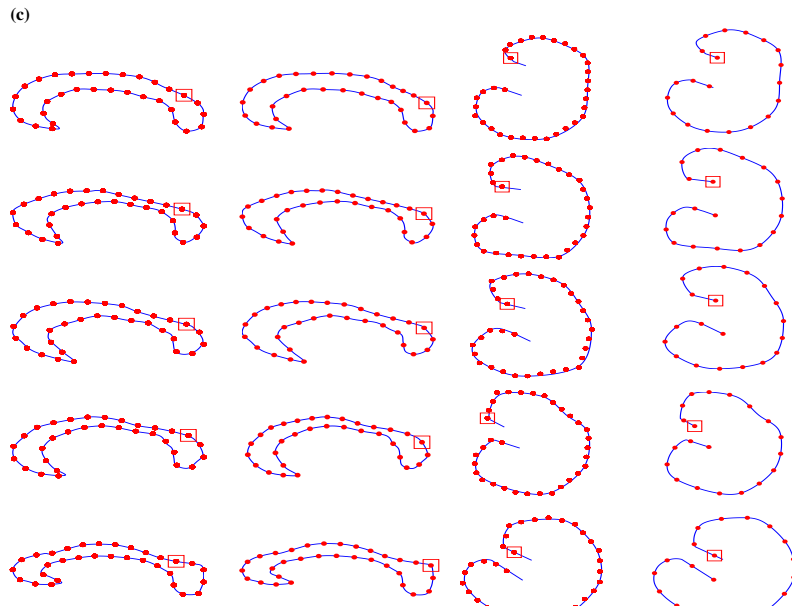

Mean Representation Error versus Number of Landmark Points
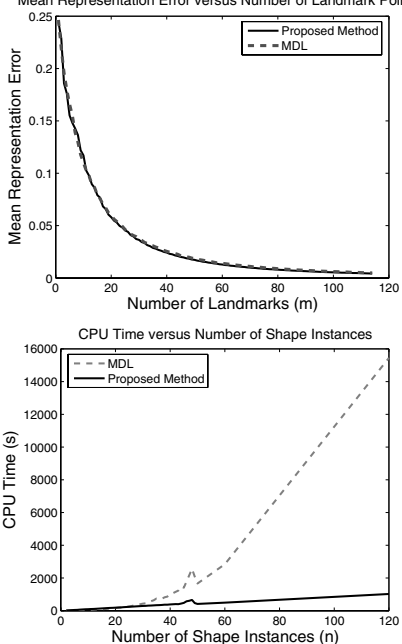

Fig. 1. (a) Illustration of shape representation error, (b) select shape instances from D1 to D5 respectively with landmarks resulting from the proposed method, and (c) selected correspondence results on D1 (corpus callosum) and D4 (kidney) with MDL results on the left and results from the proposed method on the right. See Tables 1 and 2 for statistical results on D1 and D4. Both graphs indicate further experiments in representation error and CPU time on D1. 
where $R\left(S_{i}\right)$ indicates the area enclosed by the shape contour $S_{i}$. The normalization keeps this measure invariant to the shape size.

Thin-plate models [7] have been widely used for biological shape analysis for its capability of describing nonrigid shape deformations [2]. In this paper, we use the thin-plate bending energy to model the landmark-correspondence error. Specifically, we calculate the mean shape $V_{T}=\frac{1}{n} \sum_{i=1}^{n} V_{i}$ as the template and the landmark-correspondence error from $V_{q}$ is defined as the thin-plate bending energy [7] from $V_{T}$ to $V_{q}$, i.e.,

$$
\beta\left(V_{T} \rightarrow V_{q}\right)=\frac{1}{8 \pi}\left(\mathbf{x}_{q}^{T} \mathbf{M}_{T} \mathbf{x}_{q}+\mathbf{y}_{q}^{T} \mathbf{M}_{T} \mathbf{y}_{q}\right)
$$

where $\mathbf{x}_{q}$ and $\mathbf{y}_{q}$ are columnized vectors of $x$ - and $y$-coordinates of landmarks in $V_{q}$, and $\mathbf{M}_{T}$ is the bending matrix calculated from $V_{T}$ [7]. One important property of the thin-plate bending energy is its invariance to affine transforms. The total landmark-based correspondence error can thus be defined as $\sum_{i=1}^{n} \beta\left(V_{T} \rightarrow\right.$ $V_{i}$ ). Since the landmark sets $V_{i}, i=1,2, \ldots, n$ are continually updated in our algorithm, the template shape also needs to update accordingly.

Combining these three factors, we define the shape-correspondence problem as identifying a set of $m$ landmarks $V_{i}$ from the shape contours $S_{i}, i=1,2, \ldots, n$ such that: (a) $\sum_{i} \beta\left(V_{T} \rightarrow V_{i}\right)$ is minimized; (b) $\alpha\left(V_{i}, S_{i}\right) \leq \epsilon$ for $i=1,2, \ldots, n$, where $\epsilon$ is a preset allowed representation error; (c) $m$, the number of landmarks in $V_{i}$, is minimized given condition (b) is satisfied. In the next section, we develop an algorithm to achieve these goals.

\section{Algorithm}

To solve the problem formulated in Section 2, we propose an algorithm that combines three operations: landmark sliding, landmark insertion, and landmark deletion. To start the algorithm, we perform a landmark initialization that aims to find an initial estimate of $V_{i}, i=1,2, \ldots, n$. There are many ways to achieve an initial rough landmark-based correspondence, like those successfully used for shape recognition and retrieval 41]. In this paper, we use the initialization method in 11]: uniformly sampling each shape instance into the same number of landmarks and then finding the matching across them by minimizing the thin-plate bending energy.

\subsection{Landmark Sliding}

In the landmark sliding, we slide the landmarks in $V_{q}$ along the shape contour $S_{q}$ so that the landmark-correspondence error with the template landmark set $V_{T}$ is minimized. The sliding operation consists of two steps: sliding and projection. In the sliding step, all the landmarks in $V_{q}$ are moved along the tangent directions of $S_{q}$ so that the resultant landmarks $V_{q}^{\prime}$ have the minimal landmarkcorrespondence error $\beta\left(V_{T} \rightarrow V_{q}^{\prime}\right)$. Let $\mathbf{r}_{q k}$ be the unit tangent direction at the landmark $\mathbf{v}_{q k}$, then we have 


$$
\mathbf{v}_{q k}^{\prime}=\mathbf{v}_{q k}+\gamma_{q k} \cdot \mathbf{r}_{q k}
$$

where $\mathbf{v}_{q k}^{\prime}$ is the $k$-th landmark after sliding and $\gamma_{q k}$ is the sliding distance which we want to find. Note that $\mathbf{v}_{q k}^{\prime}$ is usually not located on the shape contour $S_{q}$.

In the projection step, the landmarks $\mathbf{v}_{q k}^{\prime}, k=1,2, \ldots, m$ are projected back to $S_{q}$ to construct a new version of $V_{q}$ along $S_{q}$. This projection is achieved by updating the arc-length parameters for all landmarks:

$$
t_{q k}^{(n e w)} \leftarrow t_{q k}+\gamma_{q k}
$$

One important problem here is to preserve the shape topology, i.e., no landmark is allowed to move across its neighbors along the underlying shape contour. Therefore, we have the constraint on the sliding distance $\gamma_{q k}, k=1,2, \ldots, m$,

$$
\left(t_{q, k+1}-t_{q, k}\right) \mid L_{q}-\gamma_{q k}+\gamma_{q, k+1}>0, k=1,2, \ldots, m .
$$

The $(m+1)$-th landmark is the same as the first landmark and $a \mid b$ is the modulus operation; $\left(t_{q, k+1}-t_{q, k}\right) \mid L_{q}$ represents the traversed distance $\mathbf{v}_{q, k}$ to $\mathbf{v}_{q, k+1}$.

From these, we can see that the sliding distance $\gamma_{q k}, k=1,2, \ldots, m$, should minimize the landmark-correspondence error $\beta\left(V_{T} \rightarrow V_{q}^{\prime}\right)$, subject to the linear constraints of Eqs. (1) and (3). This is a classical quadratic-programming problem that can be effectively solved.

\subsection{Landmark Insertion and Deletion}

Both the landmark-initialization and the landmark-sliding operations have no guarantee that the obtained landmark set $V_{q}$ can represent the shape contour $S_{q}$ within the allowed representation error $\epsilon$. We address this problem by a landmark-insertion operation: If the shape-representation error $\alpha\left(V_{q}, S_{q}\right)$ is larger than the allowed threshold $\epsilon$, we simply insert an additional landmark point at $\mathbf{s}_{q}\left(0.5 \cdot\left(t_{q k}+t_{q, k+1}\right)\right)$, i.e., halfway between $\mathbf{v}_{q k}$ and $\mathbf{v}_{q, k+1}$, which contributes most to the total representation error. To keep the correspondence across shape instances, we insert an additional landmark along each of other shape instances, including the template, i.e., inserting landmark $\mathbf{s}_{i}(0.5$. $\left.\left(t_{i k}+t_{i, k+1}\right)\right)$ for each shape $S_{i}$. We repeat this landmark-insertion operation until all the shape instances are represented within the allowed error threshold $\epsilon$.

Landmark deletion is the inverse process of landmark insertion that is used to improve the representation compactness and avoid the over-sampling of the shape instances. The basic idea is to delete $k$-th landmark from all the $n$ shape instances (including the template), if the remaining landmarks can still represent all these $n$-shape instances within the allowed error threshold $\epsilon$. In practice, we in fact set a larger allowed threshold $\epsilon_{H}$ for landmark insertion and a lower threshold $\epsilon_{L}$ for landmark deletion to reduce oscillation of the iterations and improve algorithm speed. Combining all the operations, the proposed shapecorrespondence algorithm can be summarized as: 


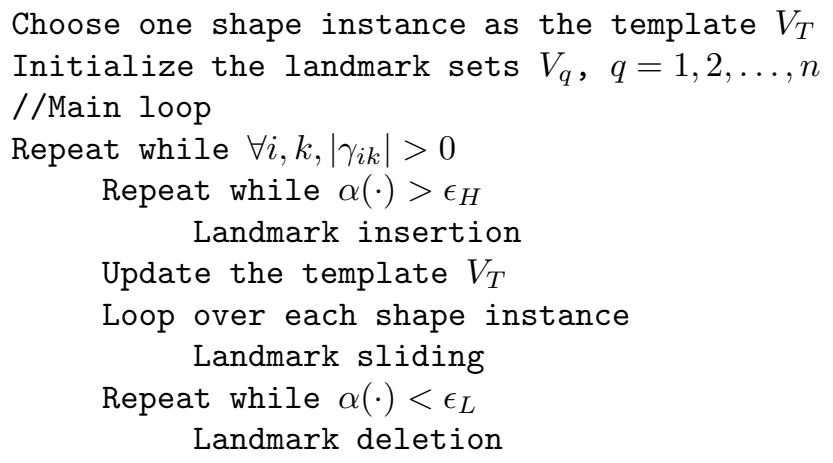

End

The stop condition of this algorithm is the convergence of all three operations, i.e., $\left|\gamma_{i k}\right|=0$ and $\epsilon_{L}<\alpha\left(V_{i}, S_{i}\right)<\epsilon_{H}, \forall i, k$. In practice, we stop the algorithm when all $\left|\gamma_{i k}\right|$ 's are sufficiently small.

\section{Experiments}

We implement the proposed method in Matlab and test it on five data sets extracted from medical images. These five data sets are: (D1) 120 corpus callosum shape instances; (D2) 24 metacarpal shape instances [10; (D3) 50 cardiac shape instances; (D4) 50 kidney shape instances; and (D5) 32 femur shape instances 10, as shown in Fig. 1(b). Among them, D1 and D2 have closed-curve shapes, D3, D4, and D5 have open-curve shapes. For comparison, we choose the MDL implementation (also in Matlab) by Thodberg [9] and, in all experiments, MDL was run with 8 active nodes optimized over 40 passes. We test MDL on each data set with three settings where the number of landmarks along each shape instance is set to 16,32 , and 64 , respectively. The proposed method is also run with three settings where the allowed representation error is set to match the average representation errors $E(\alpha)$ from the respective MDL runs $\left(\epsilon_{L}=E(\alpha)-s t d(\alpha)\right.$, $\left.\epsilon_{H}=E(\alpha)+\operatorname{std}(\alpha)\right)$. With similar representation error, we compare several other error/accuracy measures for MDL and the proposed method.

While it is very difficult to have an objective and comprehensive evaluation of the shape-correspondence performance, some quantitative measures have been developed in recent years. In this experiment, we compare the following measures: (a) $E(\beta)$ and $\operatorname{std}(\beta)$ : the mean and standard deviation of the thin-plate bending energy between the template and all the shape instances according to the identified landmarks; (b) $\lambda_{1}, \lambda_{2}$, and $\lambda_{3}$ : the three principal eigenvalues of the covariance matrix of $V_{i}, i=1,2, \ldots, n$. In calculating the covariance matrix, the Procrustes analysis [2] is applied to normalize the size and orientation of all the shape instances. (c) $m$ : the number of landmarks sampled in each shape instance, and (d) the total CPU time used for processing each data set, based on the specific implementations. In general, with a similar representation error, a good shape correspondence is expected to have small $E(\beta), \lambda_{1}, \lambda_{2}, \lambda_{3}$, and $m$. 
Table 1. Experimental results on D1(corpus callosum)

\begin{tabular}{||c||c|c|c||c|c|c||}
\hline \hline Measures & $m=16$ & $m=32$ & $m=64$ & $\begin{array}{c}\text { Mroposed } \\
\text { Method } \\
\epsilon_{L}=0.1880 \\
\epsilon_{H}=0.2669\end{array}$ & $\begin{array}{c}\text { Proposed } \\
\text { Method } \\
\epsilon_{L}=0.0199 \\
\epsilon_{H}=0.1442\end{array}$ & $\begin{array}{c}\text { Proposed } \\
\text { Method } \\
\epsilon_{L}=0.0090 \\
\epsilon_{H}=0.0885\end{array}$ \\
\hline$E(\alpha)$ & 0.2275 & 0.0819 & 0.0487 & 0.2214 & 0.0637 & 0.0164 \\
\hline$s t d(\alpha)$ & 0.0394 & 0.0620 & 0.0397 & 0.0290 & 0.0090 & 0.0023 \\
\hline$E(\beta)$ & 1.0767 & 2.0420 & 2.4937 & 0.0661 & 0.2270 & 0.4063 \\
\hline$s t d(\beta)$ & 1.3434 & 1.8201 & 1.9548 & 0.0425 & 0.1509 & 0.1490 \\
\hline$\lambda_{1}$ & 0.0159 & 0.0155 & 0.0024 & 0.0150 & 0.0093 & 0.0016 \\
\hline$\lambda_{2}$ & 0.0016 & 0.0014 & 0.0017 & 0.0025 & 0.0016 & 0.0009 \\
\hline$\lambda_{3}$ & 0.0009 & 0.0010 & 0.0004 & 0.0014 & 0.0014 & 0.0004 \\
\hline$m$ & 16 & 32 & 64 & 16 & 32 & 64 \\
\hline CPU time $(s)$ & 1073.6 & 3853.0 & 15448.0 & 340.2 & 541.4 & 1027.8 \\
\hline \hline
\end{tabular}

Table 2. Experimental results on D4(kidney)

\begin{tabular}{||c||c|c|c||c|c|c||}
\hline \hline Measures & $m=17$ & $m=33$ & $m=65$ & $\begin{array}{c}\text { Mroposed } \\
\text { Method } \\
\epsilon_{L}=0.0501 \\
\epsilon_{H}=0.0977\end{array}$ & $\begin{array}{c}\text { Proposed } \\
\text { Method } \\
\epsilon_{L}=0.0331 \\
\epsilon_{H}=0.0764\end{array}$ & $\begin{array}{c}\text { Proposed } \\
\text { Method } \\
\epsilon_{L}=0.0194 \\
\epsilon_{H}=0.0663\end{array}$ \\
\hline \hline$E(\alpha)$ & 0.0739 & 0.0547 & 0.0428 & 0.0731 & 0.0366 & 0.0142 \\
\hline$s t d(\alpha)$ & 0.0238 & 0.0216 & 0.0234 & 0.0089 & 0.0045 & 0.0020 \\
\hline$E(\beta)$ & 0.4295 & 0.9501 & 1.4099 & 0.1099 & 0.1470 & 0.2217 \\
\hline$s t d(\beta)$ & 0.1450 & 0.2841 & 0.3627 & 0.0654 & 0.1441 & 0.0992 \\
\hline$\lambda_{1}$ & 0.0031 & 0.0029 & 0.0028 & 0.0025 & 0.0024 & 0.0022 \\
\hline$\lambda_{2}$ & 0.0014 & 0.0014 & 0.0012 & 0.0015 & 0.0016 & 0.0016 \\
\hline$\lambda_{3}$ & 0.0010 & 0.0009 & 0.0008 & 0.0012 & 0.0012 & 0.0009 \\
\hline$m$ & 17 & 33 & 65 & 14 & 20 & 34 \\
\hline CPU time $(s)$ & 298.6 & 764.3 & 1396.2 & 140.4 & 211.1 & 294.8 \\
\hline \hline
\end{tabular}

For conserving space, we only show the experimental results on D1 and D4 in Tables 1 and 2. Figure 1(c) shows the identified landmarks along several sample shape instances. We can see that, given similar representation error, the proposed method produces much smaller mean bending energy $E(\beta)$ than MDL. This is reasonable since minimizing the bending energy is one of our explicit goals. An interesting result is that, in all the experiments, the proposed method produces a correspondence with $\lambda_{1}, \lambda_{2}$, and $\lambda_{3}$ that are comparable to MDL (see Table 1), because minimizing the eigenvalues of the covariance matrix is a goal of MDL but not of the proposed method. In addition, we found that the proposed method usually runs faster than MDL, especially when the number of the shape instances increases, as in the bottom graph of Fig. 1(c). For the representation compactness, we found MDL and the proposed method sample each shape instance with similar numbers of landmarks given a similar representation error as 
in the top graph of Fig. 1(c). However, in the MDL implementation, $m$, the number of landmarks, is predetermined and kept unchanged in the algorithm, while in the proposed method, $m$ is automatically determined by landmark insertion and deletion. We observed similar results on D2, D3, and D5.

\section{Conclusion}

In this paper, we developed a new landmark-based method for nonrigid shape correspondence, a prerequisite of accurate statistical shape analysis (SSA). This method considers three important factors in modelling the shape-correspondence error: landmark-correspondence error, representation error, and representation compactness. These three factors are explicitly handled by the landmark sliding, insertion, and deletion operations, respectively. The performance of the proposed method was evaluated on five shape-data sets that are extracted from medical images and the results were quantitatively compared with an implementation of the MDL method. We found that, within a similar allowed representation error, the proposed method has a performance that is comparable to or better than MDL in terms of (a) average bending energy, (b) principal variances in SSA, (c) representation compactness, and (d) algorithm speed.

\section{References}

1. S. Belongie, J. Malik, and J. Puzicha. Shape matching and object recognition using shape contexts. IEEE Trans. PAMI, 24(24):509-522, April 2002.

2. F. L. Bookstein. Principal warps: Thin-plate splines and the decomposition of deformations. IEEE Trans. PAMI, 11(6):567-585, June 1989.

3. F. L. Bookstein. Landmark methods for forms without landmarks: Morphometrics of group differences in outline shape. Medical Image Analysis, 1(3):225-243, 1997.

4. H. Chui and A. Rangarajan. A new algorithm for non-rigid point matching. In Proc. Conf. Computer Vision and Pattern Recog., pages 44-51, 2000.

5. T. F. Cootes, C. J. Taylor, D. H. Cooper, and J. Graham. Active shape models their training and application. Comput. Vision Image Understanding, 61(1):38-59, Jan. 1995.

6. R. Davies, C. Twining, T. Cootes, J. Waterton, and C. Taylor. A minimum description length approach to statistical shape modeling. IEEE Trans. Med. Imag., 21(5):525-537, May 2002.

7. J. Duchon. Splines minimizing rotation-invariant semi-norms in sobolev space. In Constructive Theory of Functions of Several Variables, Lecture Notes in Mathematics, 571, pages 85-100, 1977.

8. C. G. Small. The Statistical Theory of Shape. Springer-Verlag, 1996.

9. H. H. Thodberg. Adding curvature to minimum description length shape models. In British Machine Vision Conference, volume 2, pages 251-260, 2003.

10. H. H. Thodberg. Minimum description length shape and appearance models. In IPMI, pages 51-62, 2003.

11. S. Wang, T. Kubota, and T. Richardson. Shape correspondence through landmark sliding. In Proc. Conf. Computer Vision and Pattern Recog., pages 143-150, 2004. 